\title{
A semiempirical approach to a viscously damped oscillating sphere
}

\author{
P Alexander ${ }^{1}$ and E Indelicato ${ }^{2}$ \\ ${ }^{1}$ Departamento de Física, Facultad de Ciencias Exactas y Naturales, Universidad de Buenos \\ Aires, Argentina \\ ${ }^{2}$ Laboratorio de Mecánica Elemental, Departamento de Física, Facultad de Ciencias Exactas y \\ Naturales, Universidad de Buenos Aires, Argentina \\ E-mail: peter@df.uba.ar
}

Received 9 March 2004, in final form 17 August 2004

Published 26 October 2004

Online at stacks.iop.org/EJP/26/1

\begin{abstract}
A simple model of damped harmonic motion is usually presented in undergraduate physics textbooks and straightforwardly applied for a variety of well-known experiments in student laboratories. Results for the decaying vertical oscillation of a sphere attached to the lower end of a spring in containers with different liquids are analysed here under this standard framework. Some important mismatches between observation and theory are found, which are attributed to oversimplifications in the formulation of the drag force. A more elaborate expression for the latter within a semiempirical approach is then introduced and a more appropriate description of the measurements is shown to be attained. Two coefficients account for experimental corrections, which under certain conditions permit in addition the calculation of specific fluid quantities associated with the oscillating sphere. Rough relations between viscosity and damping factor under appropriate limits are derived. The laboratory experience may also be used to introduce the concept of a semiempirical model and exhibit its utility in physics.
\end{abstract}

\section{Introduction}

Linear viscous damping (drag force term proportional to the first power of velocity) on a body possesses an interesting characteristic: exact solutions to the resulting differential equation of motion may be obtained by elementary mathematical methods and they are easy to interpret. The pedagogical importance of the scheme due to its appealing simplicity is clear. However, it is often an inadequate representation of reality. It then becomes essential to check that the conditions of an experiment satisfy the validity ranges that imply linearity, which are rather restrictive and rarely met in practice. Moreover, empirical versions of the ideal model are apparently quite difficult to yield [1-8]. 
This paper refers to an experiment that meets the stringent requisites for linear damping (if appropriate conditions are chosen, see section 4): the vertical displacement of a sphere suspended from a spring and submerged in a viscous fluid. Weight, upthrust, elastic and drag forces are in this case present and the familiar equation for the displacement $x$ (positive upwards) of the sphere from the rest position is

$$
\ddot{x}+2 \gamma \dot{x}+\omega_{o}^{2} x=c
$$

where $c$ is a constant that produces a fixed shift in $x$ from the spring equilibrium position, so we will set it null henceforth as we are rather interested in the motion of the sphere. If $\omega_{o}^{2}>\gamma^{2}$, i.e. there is some preponderance of elastic over frictional force (at least for some time), the solution in terms of time $t$ will look like

$$
x=A \mathrm{e}^{-\gamma t} \cos (\omega t+\varphi)
$$

where $A$ is the amplitude of undamped oscillation, $\gamma$ is the damping coefficient (the exponential decay is independent of the initial amplitude due to the linear nature of the system),

$$
\omega=\sqrt{k / M-\gamma^{2}}
$$

is the oscillation frequency $\left(\omega_{o} \equiv(k / M)^{1 / 2}\right.$ is the undamped natural frequency of the sphere, where $k$ is the spring constant and $M$ is the hanging mass) and $\varphi$ is the initial phase constant (nonzero if timing does not start simultaneously with release). As $\omega$ is defined in terms of $k, M$ and $\gamma$, only three parameters remain independent $(A, \varphi$ and $\gamma$ or $\omega)$ and they may be determined from the fit of equation (2) to every experimental displacement against time curve. It is clear in this kind of experiment that the damping coefficient depends among many factors on certain fluid properties, where viscosity surely plays an important role. A drag force given by Stokes' law leads in equation (1) to

$$
\gamma=\frac{3 \pi r \eta}{M}
$$

where $\eta$ is the liquid viscosity and $r$ is the radius of the sphere.

The experimental set-up is exhibited below and results are then presented for diverse liquids. Problems emerge clearly when the appropriateness of the above standard model for the present experiment is assessed. It will be shown however, that, an analysis of the observed motion with an appropriate alternative approach may lead the students to bring observation and theory closer and to extract physically meaningful information. The present experimental work may also be helpful to introduce or reinforce in early curriculum alumni the idea of the use of a semiempirical model as a convenient tool when one deals with shortcomings of the available theory. In addition, we suggest that the experience can be used to stimulate the students for further research on a general relation between damping factor and viscosity (the procedure may be easily extended to liquids other than those considered here). It is demanding but suitable for those who attend a first course on fluid dynamics or for a mechanics syllabus including this area of physics. The necessary equipment should be available in most undergraduate mechanics laboratories.

\section{Experimental set-up and procedure}

On a tall sturdy support rod, we clamped a Pasco CI-6519 force sensor and from its hook a spring hung. We suspended an iron ball from it with an appropriately prepared thin cylindrical aluminium rod, the ball being submerged a few centimetres under the liquid surface in a measuring cylinder (figure 1) scaled up to 21 with diameter and height respectively of $(8.0 \pm$ $0.1) \mathrm{cm}$ and $(46.8 \pm 0.1) \mathrm{cm}$. The rod was used to avoid any immersion of the lowest part of the 


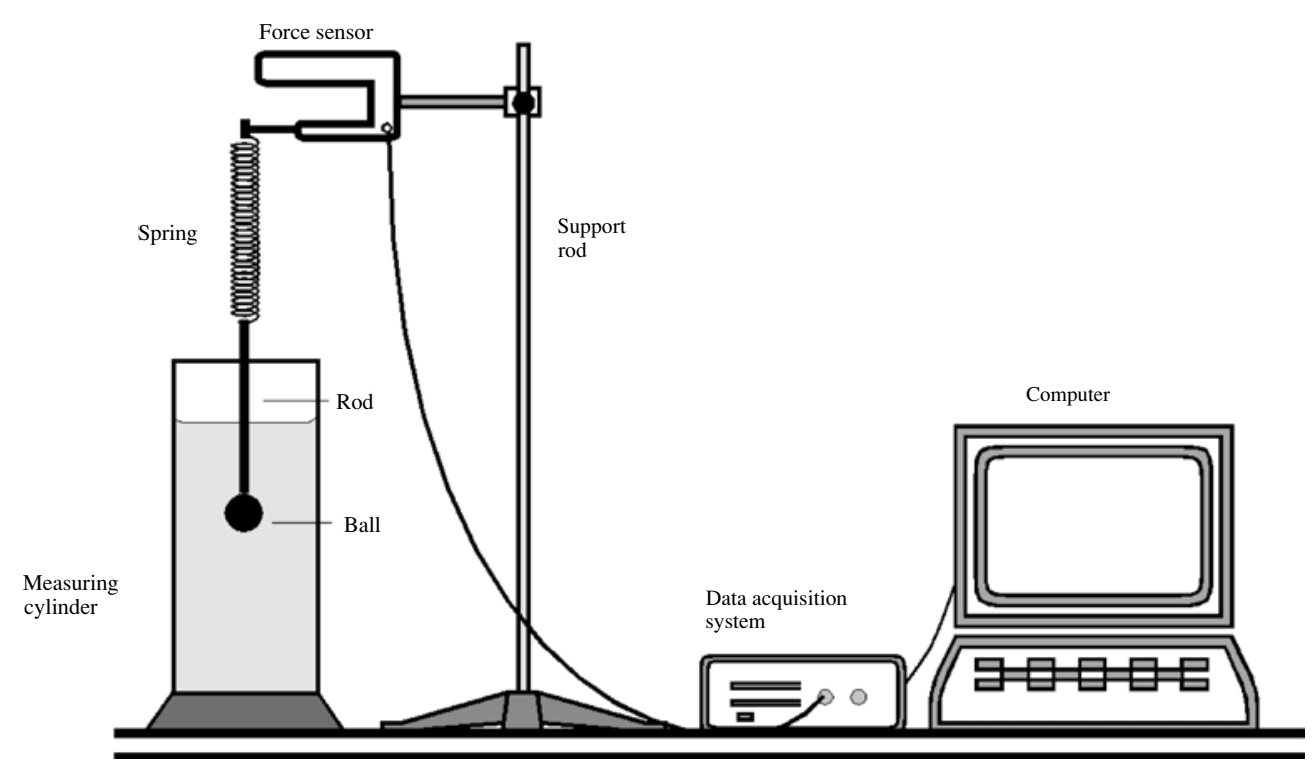

Figure 1. Schematic diagram of the experimental set-up (not all elements are to scale).

spring in the liquid. It had a small hole close to one extreme for the attachment to the spring and it had already been fastened to the ball on the opposite side. The force sensor was connected to a computer data acquisition system (MPLI), which recorded force against time. The sampling frequency was set to $50 \mathrm{~Hz}$ in a compromise between time resolution and recording span availability. A too large frequency would not only yield a very short observation time interval but could also introduce uninteresting high frequency noise. We initiated free oscillations of the system in the vertical plane with a small displacement of the rod either upwards or downwards. We had to ensure that the deflections were one-dimensional and small, i.e. the displacement amplitude had to be smaller than the sphere diameter. Measurement start and system release were not necessarily simultaneous.

We have previously checked that there was a linear relation between spring force and displacement and we determined the slope in order to convert the measurements of the former quantity into values of the latter. This permitted us to display the curve displacement against time and then find out the values of the parameters from the corresponding fit. The effective oscillating spring mass fraction $e$ was also determined, so we were able to calculate the total oscillating mass $M=m_{b}+e m_{s}+m_{r}$, where $m_{b}, m_{s}$ and $m_{r}$ refer to the ball, spring and rod mass, respectively. A few variations of the experiment conditions have been conducted for this laboratory experience. We used tap water, mineral (SAE 40) and vegetal (sunflower) oil. The values of diverse quantities that are important for the experiment may be found in table 1 .

\section{Results of the standard model}

Figure 2 represents displacement from the equilibrium position against time data when the sphere was immersed in the measuring cylinder with, respectively, water, vegetal and mineral oil and the parameters of the corresponding fits have been summarized in table 2. It may be seen that there are some graphical discrepancies mainly due to inadequate $\omega$ and $\gamma$. The model 
Table 1. Quantities relevant to the experiment.

\begin{tabular}{ll}
\hline$k\left(\mathrm{~N} \mathrm{~m}^{-1}\right)$ & $27.9 \pm 0.1$ \\
$e$ & $0.379 \pm 0.006$ \\
$m_{\mathrm{s}}(g)$ & $62.8 \pm 0.1$ \\
$m_{r}(\mathrm{~g})$ & $20.1 \pm 0.1$ \\
$m_{b}(\mathrm{~g})$ & $106.2 \pm 0.1$ \\
$r(\mathrm{~cm})$ & $1.50 \pm 0.01$ \\
$\eta_{w}(P)^{\mathrm{a}}$ & $0.00915 \pm 0.00010$ \\
$\eta_{v}(P)^{\mathrm{a}}$ & $0.56 \pm 0.01$ \\
$\eta_{m}(\mathrm{P})^{\mathrm{a}}$ & $3.6 \pm 0.1$ \\
$\rho_{w}\left(\mathrm{~g} \mathrm{~cm}^{-3}\right)^{\mathrm{a}}$ & $0.997 \pm 0.001$ \\
$\rho_{v}\left(\mathrm{~g} \mathrm{~cm}^{-3}\right)^{\mathrm{a}}$ & $0.918 \pm 0.001$ \\
$\rho_{m}\left(\mathrm{~g} \mathrm{~cm}^{-3}\right)^{\mathrm{a}}$ & $0.859 \pm 0.001$
\end{tabular}
a The values correspond to a temperature of $(23.5 \pm 0.5){ }^{\circ} \mathrm{C}$. Subindices $w, v$ and $m$ correspond
respectively to water, vegetal and mineral oil.

Table 2. Parameter values according to fits of the standard model.

\begin{tabular}{llll}
\hline & Water & Vegetal oil & Mineral oil \\
\hline$A(\mathrm{~cm})$ & $0.41 \pm 0.02$ & $0.67 \pm 0.03$ & $0.29 \pm 0.01$ \\
$\gamma\left(\mathrm{s}^{-1}\right)$ & $0.14 \pm 0.01$ & $0.87 \pm 0.05$ & $1.86 \pm 0.10$ \\
$\varphi(\mathrm{rad})$ & $2.14 \pm 0.02$ & $0.00 \pm 0.03$ & $0.29 \pm 0.03$
\end{tabular}

Correlation coefficients $r^{2}$ for the fits ranged between 0.8 and 0.9 .

predicts nearly the same oscillation frequency for the three fluids. According to equation (3) $\omega$ depends on $\omega_{o}$ and $\gamma$, where the former parameter is much larger than the second quantity in our cases $\left(\omega_{o} \approx 13.6 \mathrm{~s}^{-1}, \gamma \sim 1 \mathrm{~s}^{-1}\right)$. Then $\omega$ is essentially given by $\omega_{o}$ and therefore its dependence on $\eta$ (through $\gamma$ ) is very weak. In addition, the damping coefficients obtained from the experiments lead in equation (4) to order of magnitude wrong viscosity values: (1.44 \pm $0.04) \mathrm{P},(9.23 \pm 0.21) \mathrm{P}$ and $(19.8 \pm 0.9) \mathrm{P}$, respectively, for water, vegetal and mineral oil (compare with table 1). Something must be wrong; intuition suggests the use of a generalization of Stokes' law: drag force proportional to velocity and viscosity through some unknown constant, so $\eta \sim \gamma$. It is then very tempting to use a decaying oscillating body in diverse liquids to obtain the relative viscosities from the ratios of the corresponding damping factors [9], but this procedure is not correct. In fact, the values so derived are here in strong disagreement with those that may be calculated from table 1.

Is it then possible to overcome the above difficulties? Some important aspects may have been omitted in the standard model. This justifies the introduction of a more elaborate description of the resistance force on the sphere than Stokes' law, and it will also be necessary to recall some specific fluid concepts.

\section{An alternative approach}

Linear damping is valid only for $\mathrm{Re} \leqslant 1$ in the case of a sphere with uniform motion. Some articles and textbooks have applied this law straightforwardly even at large Re with no substantiation on its application. A damping term proportional to velocity has been observed by experimental means [1,6] for oscillating bodies at large Re, but according to theory three conditions should be met $[10,11]$ : the flow should not separate from the solid boundary and the characteristic length of the body must be much larger than the oscillation amplitude 

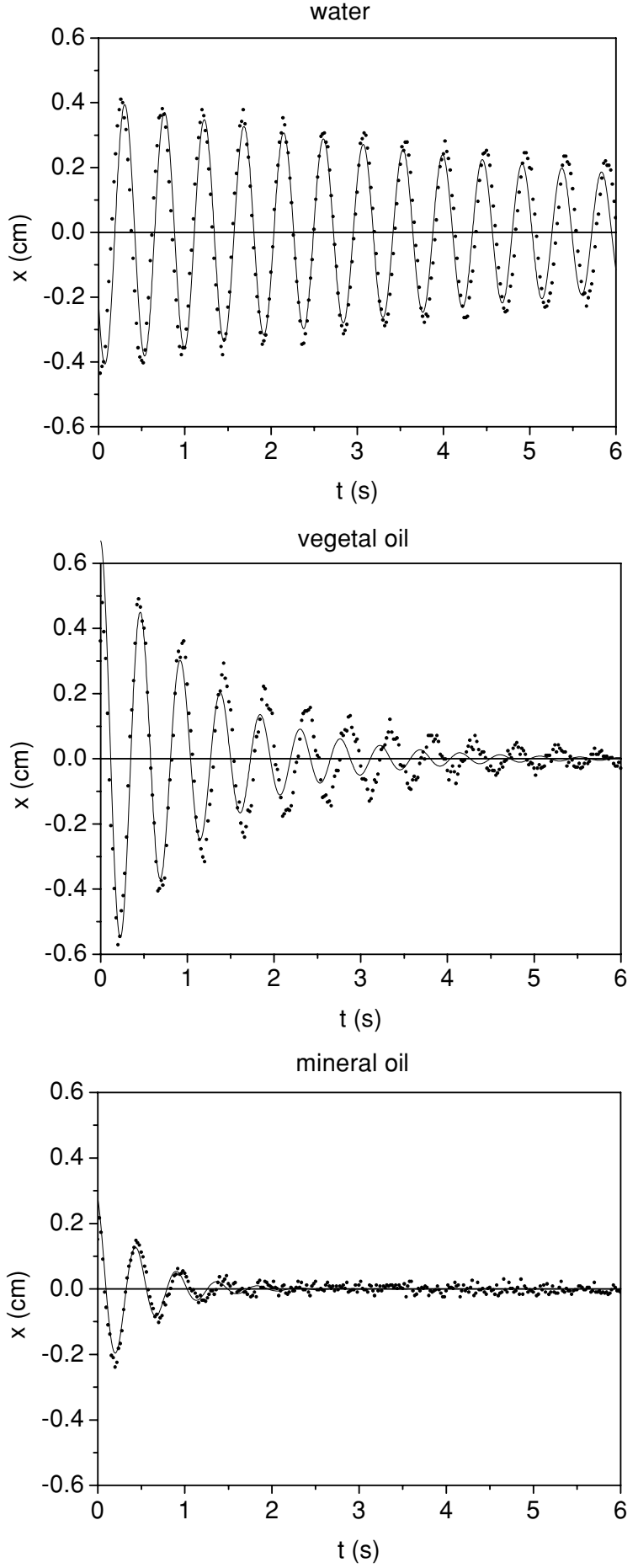

Figure 2. Displacement against time data (dots) for the sphere immersed in the measuring cylinder with different liquids. Fits (solid curves) according to the standard model are also shown. 
and the penetration depth (to be defined below). It is extremely unlikely that the last two conditions will be met (which reasonably happens in the present experiments) without the first one [6]. Stokes' law leads to wrong results when it is applied to the present experiment, but there are obviously many more linear damping options available. Two possible sources for improvements are the incorporation of a more appropriate proportionality factor between drag force and velocity and the contemplation of the liquid mass accompanying the sphere motion. We discuss this in the following paragraphs.

A large amount of experimental and theoretical work has been done on the drag force on a body moving in a fluid. The formulae for a sphere undergoing uniform motion in the low and large Reynolds number $(\mathrm{Re})$ limits are well known, being, respectively, proportional to the first (Stokes law) and second power of its velocity $\mathbf{v}$. The expression for a sphere oscillating in an incompressible fluid for small amplitude of oscillation with respect to the diameter $2 r$ is $[10,12]$

$$
\mathbf{F}=-\left[6 \pi \eta r\left(1+\frac{r}{\delta}\right) \mathbf{v}+3 \pi r^{2}\left(1+\frac{2}{9} \frac{r}{\delta}\right) \rho \delta \frac{\mathrm{d} \mathbf{v}}{\mathrm{d} t}\right]
$$

where $\mathbf{F}$ is the drag force,

$$
\delta=\sqrt{\frac{2 \eta}{\rho \omega}}
$$

is defined as the penetration depth and is used as an estimation for the thickness of the boundary layer around the sphere, where $\rho$ denotes the density of the fluid. The only restriction on $\operatorname{Re}=2 r \mathrm{v} \rho / \eta$ is that it must be much larger than the ratio of oscillating amplitude and diameter. The expressions accompanying the steady flow Stokes law in equation (5) are called history and added mass terms and they approach zero for null oscillation frequency. If $r / \delta \gg 1$ (in the second term $\gg$ means at least 40 ), which is roughly equivalent to $\operatorname{Re} \gg 1$ [11], we obtain

$$
\mathbf{F}=-\left[\frac{6 \pi r^{2} \eta}{\delta} \mathbf{v}+\frac{2}{3} \pi r^{3} \rho \frac{\mathrm{d} \mathbf{v}}{\mathrm{d} t}\right]
$$

where the second term represents the added mass (one half of the liquid displaced is the amount supposed to accompany the sphere motion) in an incompressible ideal fluid or in the large frequency limit if viscosity is included [10].

Some coarse approximations may be found in the derivation of equation (5). In order to optimize its application to each case below, experimental correction factors will be used. This practice has spawned several semiempirical equations, which are commonly used in diverse applications. We will concentrate on two potential adjustments. In the first and second terms of equation (5), we will respectively introduce empirical coefficients $f_{1}$ and $f_{2}$, so

$$
\mathbf{F}=-\left[\frac{6 \pi \eta r}{f_{1}}\left(1+\frac{r}{\delta}\right) \mathbf{v}+f_{2} 3 \pi r^{2}\left(1+\frac{2}{9} \frac{r}{\delta}\right) \rho \delta \frac{\mathrm{d} \mathbf{v}}{\mathrm{d} t}\right] .
$$

We should note that both factors have a simple and straightforward meaning when $r / \delta \gg 1$ :

$$
\mathbf{F}=-\left[\frac{6 \pi r^{2} \eta}{f_{1} \delta} \mathbf{v}+f_{2} C \frac{4}{3} \pi r^{3} \rho \frac{\mathrm{d} \mathbf{v}}{\mathrm{d} t}\right]
$$

so $f_{1}$ considers any difference in the estimation of the boundary layer thickness by the penetration depth [11] and $f_{2}$ accounts for the deviation in the experiment from the coefficient $C=1 / 2$ deduced by ideal or viscous theory for the added mass of a sphere as mentioned above. Values about 1 should be expected for $f_{1}$ and $f_{2}$ if the experimental conditions approximately suit the validity ranges. 
Table 3. Parameter values according to fits of the semiempirical model.

\begin{tabular}{|c|c|c|c|c|c|c|}
\hline & \multicolumn{3}{|c|}{ Measuring cylinder } & \multicolumn{3}{|c|}{ Can } \\
\hline & Water & Vegetal oil & Mineral oil & Water & Vegetal oil & Mineral oil \\
\hline$A(\mathrm{~cm})$ & $0.421 \pm 0.001$ & $0.591 \pm 0.001$ & $0.290 \pm 0.001$ & $0.701 \pm 0.001$ & $0.710 \pm 0.001$ & $0.298 \pm 0.001$ \\
\hline$\gamma\left(\mathrm{s}^{-1}\right)$ & $0.111 \pm 0.002$ & $0.564 \pm 0.009$ & $1.67 \pm 0.06$ & $0.079 \pm 0.001$ & $0.516 \pm 0.008$ & $1.56 \pm 0.02$ \\
\hline$\omega\left(\operatorname{rad~s}^{-1}\right)$ & $13.39 \pm 0.01$ & $13.03 \pm 0.01$ & $12.53 \pm 0.05$ & $13.42 \pm 0.01$ & $13.10 \pm 0.01$ & $12.52 \pm 0.02$ \\
\hline$\varphi(\mathrm{rad})$ & $2.696 \pm 0.005$ & $0.340 \pm 0.010$ & $0.550 \pm 0.020$ & $0.884 \pm 0.004$ & $3.900 \pm 0.010$ & $3.320 \pm 0.010$ \\
\hline
\end{tabular}

Correlation coefficients $r^{2}$ for the fits were all above 0.95 .

Table 4. Experimental determination of correction factors of the semiempirical model.

\begin{tabular}{|c|c|c|c|c|c|c|}
\hline & \multicolumn{3}{|c|}{ Measuring cylinder } & \multicolumn{3}{|c|}{ Can } \\
\hline & Water & Vegetal oil & Mineral oil & Water & Vegetal oil & Mineral oil \\
\hline$f_{1}$ & $0.31 \pm 0.02$ & $0.50 \pm 0.04$ & $0.49 \pm 0.05$ & $0.44 \pm 0.02$ & $0.56 \pm 0.04$ & $0.53 \pm 0.04$ \\
\hline$f_{2}$ & $0.69 \pm 0.10$ & $1.12 \pm 0.11$ & $1.17 \pm 0.13$ & $0.62 \pm 0.10$ & $0.99 \pm 0.10$ & $1.20 \pm 0.08$ \\
\hline
\end{tabular}

The equation of motion for the sphere after equation (7) becomes

$$
\ddot{x}+\frac{\frac{6 \pi r \eta}{f_{1}}\left(1+\frac{r}{\delta}\right)}{f_{2} 3 \pi r^{2}\left(1+\frac{2}{9} \frac{r}{\delta}\right) \rho \delta+M} \dot{x}+\frac{k}{f_{2} 3 \pi r^{2}\left(1+\frac{2}{9} \frac{r}{\delta}\right) \rho \delta+M} x=\frac{\left(\frac{4}{3} \pi r^{3} \rho-M\right) g}{f_{2} 3 \pi r^{2}\left(1+\frac{2}{9} \frac{r}{\delta}\right) \rho \delta+M}
$$

whereas for $r / \delta \gg 1$ it reduces to

$$
\ddot{x}+\frac{6 \pi r^{2} \eta}{f_{1} \delta\left(f_{2} C \frac{4}{3} \pi r^{3} \rho+M\right)} \dot{x}+\frac{k}{f_{2} C \frac{4}{3} \pi r^{3} \rho+M} x=\frac{\left(\frac{4}{3} \pi r^{3} \rho-M\right) g}{f_{2} C \frac{4}{3} \pi r^{3} \rho+M}
$$

where $g$ is gravity. The large fractions are all constants, so we may cast the above expressions in the same form as equation (1). However, here we have an unknown $f_{2}$, so $\omega_{o}$ cannot be calculated a priori (for example, in the expression of $\omega_{0}$ obtained from equation (9) it is not possible to clearly specify the added mass of the sphere prior to the experiment), and $\omega$ is now a fourth independent parameter.

The new fits respectively for water, vegetal and mineral oil may be found in figure 3 . An improvement with respect to figure 2 becomes clear. The calculated parameters have been summarized in table 3. It may be evaluated in equation (8) that $\omega_{o}$ within this framework mainly depends on the spring constant and the hanging mass and only marginally on the fluid properties, but this slight change gives in the calculation of $\omega$ (equation (3)) an important correction to improve the fits with respect to the standard model as it has an accumulative effect in time. Note that this better $\omega$ does not stem from a more appropriate $\gamma$ but from a more adequate $\omega_{o}$ which takes into account the fluid around the sphere. In addition, even with $f_{1}=$ $f_{2}=1$ the new approach gives more appropriate viscosities than the standard model: $(0.09 \pm$ $0.04) \mathrm{P},(1.8 \pm 0.1) \mathrm{P}$ and $(10.3 \pm 0.1) \mathrm{P}$ respectively for water, vegetal and mineral oil, which are however still far from the values in table 1.

The possible presence of wall or bottom effects was evaluated by respectively repeating these three experiments in a large can of volume around 8.51 with diameter and height respectively $(21.6 \pm 0.1) \mathrm{cm}$ and $(23.3 \pm 0.1) \mathrm{cm}$, which was almost full with each of the corresponding liquids. Some significant differences in $\gamma$ and $\omega$ were observed with respect to the measuring cylinder (see table 3), so we had to conclude that those effects were present.

We calculated the values of $f_{1}$ and $f_{2}$ from $\gamma$ and $\omega$ for each part of the experiment. The corresponding values may be found in table 4 . Water exhibited the largest deviations from the 

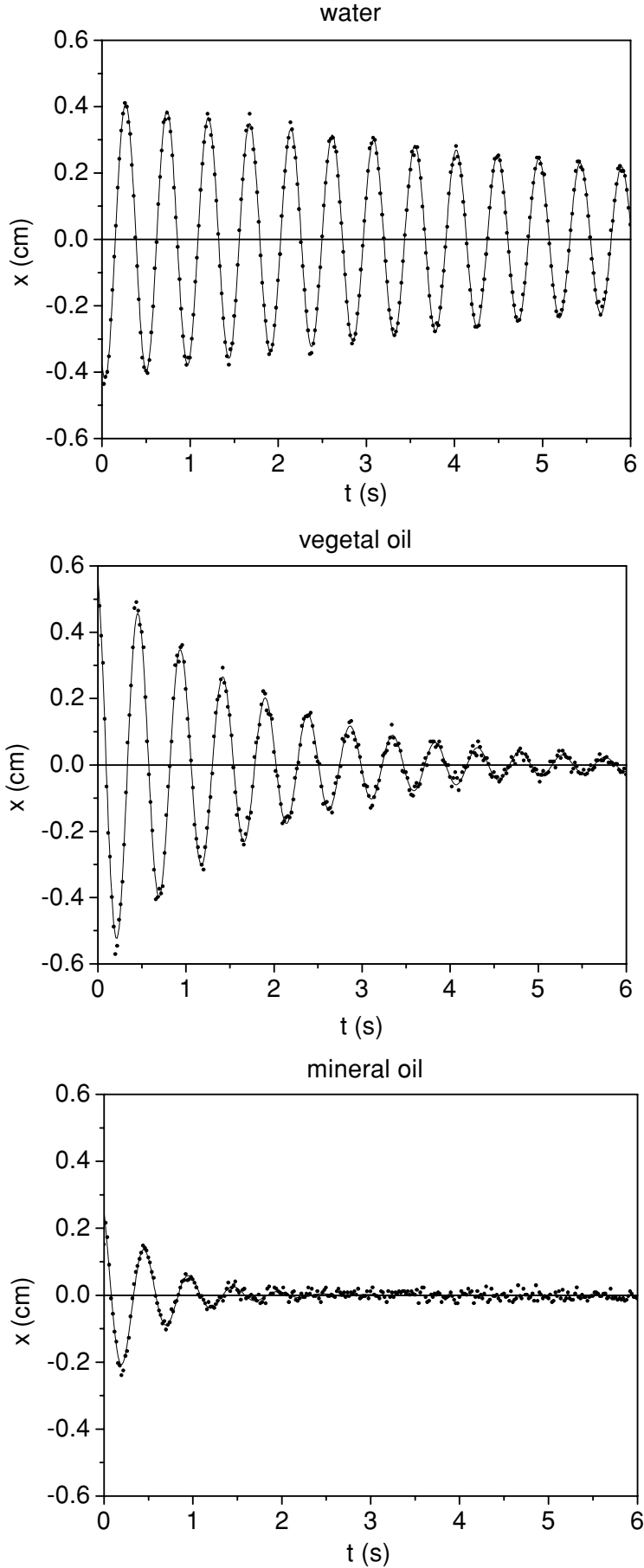

Figure 3. Displacement against time data (dots) for the sphere immersed in the measuring cylinder with different liquids. Fits (solid curves) according to the semiempirical model are also shown. 
ideal value of 1 and vegetal oil the smallest. There is a trend (although there are no significant differences in every comparison) in the bigger container for smaller $\gamma$ and larger $\omega$ and for lower $f_{2}$ and larger $f_{1}$.

In reference to a possible relation between damping and viscosity, let us visualize the second left-hand term in equation (8). For each liquid $f_{1}, f_{2}$ may be different, but always of order 1. In the denominator $M$ makes an overwhelming contribution in our experiments (typically 90\%). Considering the definition of $\delta$ in equation (6), we may then grossly infer a $\gamma \sim \eta^{1 / 2}$ law for $r / \delta \gg 1$ and $\gamma \sim \eta$ for $r / \delta \ll 1$, as $\rho$ and $\omega$ do not change significantly among the liquids used.

For clearly underdamped cases $\left(\omega \approx \omega_{0}\right)$, the validity of $r / \delta \gg 1$ may be ensured according to equation (6) by selecting the elements so that

$$
\sqrt{\frac{2 \eta}{\rho}} \ll r \sqrt[4]{k / M} .
$$

In the present experiments $r / \delta$ was of the order of 40,5 and 2, respectively, for water, vegetal and mineral oil. This implies that a dominant contribution to the drag force from the terms containing this ratio in equation (5) is expected only for water. The empirical coefficients may then be used to estimate the boundary layer thickness and the added mass coefficient of the oscillating sphere in this liquid.

As already mentioned $r / \delta \gg 1$ is roughly equivalent to $\operatorname{Re} \gg 1$, which may be proved and tested:

$$
\frac{r}{\delta} \approx \frac{r \sqrt{\omega \rho}}{\sqrt{\eta}} \approx \sqrt{\operatorname{Re}} .
$$

In fact, the Re values of the experiments for water, vegetal and mineral oil are about 1500 , 30 and 5 respectively (depending in detail upon whether one uses the radius or the diameter of the sphere or the amplitude of oscillation as a reference length) and the above expression seems to relate $r / \delta$ and Re in a quite faithful manner.

\section{Conclusions}

Besides its wide use, some failures are shown to exist in the standard model for the description of the dynamics of a viscously damped oscillating sphere in a liquid. We therefore use a more elaborate expression for the drag force to replace Stoke's law and a relatively simple semiempirical approach including two coefficients is then introduced. Changes with respect to the standard model in the dependence of the oscillating frequency and the damping on the variables of the problem emerge, which bring theory and observation closer. In particular, it is then explained that the damping factor ratio of the decaying oscillation of a body in diverse liquids may not be used to infer the corresponding relative viscosities. The results found for the two empirical correction factors in three liquids showed that their use becomes advisable in the description of oscillating spheres and the most important effect here was in water and the least in vegetal oil. If the selected experiment conditions satisfy (10), it is then possible to determine the values of two specific fluid magnitudes: the added mass coefficient and the boundary layer thickness associated with the oscillating sphere. Wall and bottom produce some small but noticeable effects on oscillating frequency and damping factor, i.e. on both empirical correction coefficients. We have shown that square root or linear law relationships between damping factor and viscosity are roughly to be expected if respectively condition (10) or its opposite hold. We encourage students to repeat this experiment with other liquids in order to test these laws and to extend the calculations of added mass and boundary layer with configurations that suit (10). 


\section{Acknowledgments}

We wish to express our gratitude to the Physics Department for equipment availability and to laboratory staff F Buceta, O Pagani and G Loureiro for their assistance and patience in the preparation of parts and equipment for the experiments. PA is a member of Conicet.

\section{References}

[1] Gupta V K, Shanker G and Sharma N K 1986 Experiment on fluid drag and viscosity with an oscillating sphere Am. J. Phys. 54619

[2] Mellen W R 1990 Oscillation of a gas balloon due to a temperature gradient Am. J. Phys. 58781

[3] Bisquert J, Ramirez P, Barbero A J and Mafé S 1991 A classroom demonstration on air drag forces Eur. J. Phys. 12249

[4] Peters R D and Pritchett T 1997 The not-so-simple harmonic oscillator Am. J. Phys. 651067

[5] Timmerman P and van der Weele J P 1999 On the rise and fall of a ball with linear or quadratic drag Am.J. Phys. 67538

[6] Libii J N 2000 Demonstration of viscous damping in the undergraduate laboratory Am. J. Phys. 68195

[7] Wang X, Schmitt C and Payne M 2002 Oscillations with three damping effects Eur. J. Phys. 23155

[8] Mallinckrodt A J 2003 Drag forces Phys. Teach. 41261

[9] Zonetti L F C, Camargo A S S, Sartori J, de Sousa D F and Nunes L A O 1999 A demonstration of dry and viscous damping of an oscillating pendulum Eur. J. Phys. 2085

[10] Landau L D and Lifshitz E M 1959 Fluid Mechanics (New York: Pergamon)

[11] Batchelor G K 1967 An Introduction to Fluid Dynamics (Cambridge: Cambridge University Press)

[12] Lamb H 1997 Hydrodynamics (New York: Cambridge University Press) 